\title{
Web-Linkage Viewer: Drawing Links in the Web Based on a Site-Oriented Framework
}

\author{
Yasuhito Asano and Takao Nishizeki \\ Graduate School of Information Sciences, Tohoku University, \\ Aoba-yama, Sendai, Japan
}

\section{Introduction}

In recent years, link-based information retrieval methods from the Web are developed, such as HITS and Trawling. Since these methods utilize characteristic graph structures of links in the Web, analyzing the links by drawing them understandably will play an important role in the link-based information retrieval. Moreover, Asano et al. [2, 3] have shown that a framework using a site as a unit of information is more natural and useful for link-based information retrieval than the existing framework using a page as a unit. Therefore, we should distinguish links inside a site (called local-links) between links between sites (called global-links) and analyze their own graph structures. However, existing drawing tools, such as Gravis [1] and H3Viewer [5], do not distinguish locallinks between global-links, and therefore they cannot draw graph structures of these links understandably. In this paper, we propose a new drawing tool, named Web-linkage Viewer, in order to draw graph structures in the Web according to the site-oriented framework.

\section{Web-Linkage Viewer}

When a site is regarded as a unit of information as humans naturally consider so, every page must belong to some site, and then the following two kinds of links can be defined. (1) Let $A$ and $B$ be two distinct sites. If there is a link from a page $v$ in $A$ to a page $w$ in $B$, we say there is a global-link from $A$ to $B$. (2) A link from a page $v$ to a page $w$ with $v$ and $w$ in $A$ is called a local-link inside $A$.

In order to show relationships between a site and other sites and relationships between pages and sites understandably, we draw global-links and local-links distinctively in the same space and avoid overlaps of the local-links in some site with the local-links in another site as much as possible. Concretely, we draw sites as cubes and global-links as arrows on a spherical surface, and for each site, draws the pages inside the site as cubes and the local-links as lines in a cone emanating from a cube representing the site. The sites are arranged by using the spring model and the pages in each site are arranged from the apex of cone to the base according to the order determined by the breadth first search of the local-links in the site. See [2] for the details. 


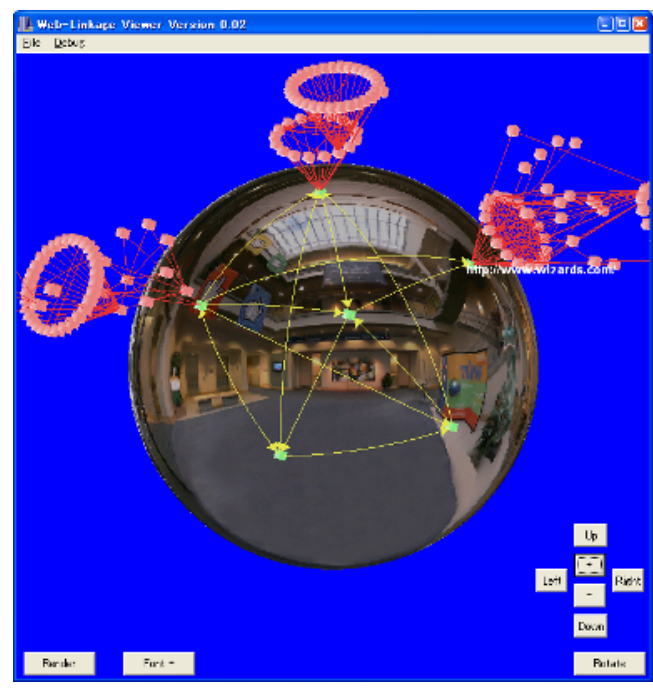

Fig.1. Screenshot of Web-linkage Viewer

By drawing the global-links and the local-links distinctively in the same space, we can understand a relationship between a site and pages inside it.

As a result, we conclude that our system provides us with a more understandable drawing of structures of links in the Web. In particular, by using our system we can understand that a large part of local-links inside each site forms a tree structure frequently (i.e. other kinds of links, such as links connecting siblings, are fewer), while global-links have another kind of structure.

We implemented our software on Microsoft Windows XP by using Borland $\mathrm{C}++$ Builder 5.0 and Microsoft DirectX 9.0a. It can zoom in and out, rotate the drawing by mouse, and display URLs of the nodes and open a page with Internet Explorer by click. We have compared our drawing with drawings of Gravis and H3Viewer and shown that our drawing is more understandable than them [2]. We have developed also a tool named Neighbor Community Finder for finding Web communities related to given URLs [4]. As a future work, we are trying to combine this tool with our viewer, in order to provide this tool with a GUI and to visualize graph structures in neighbor sites and pages of found communities.

Acknowledgments. We thank Prof. Hiroshi Imai and Ms. Sonoko Moriyama for their comments and help for computational experiments of comparison of our drawing with other tools.

\section{References}

1. Gravis. http://www-pr.informatik. uni-tuebingen.de/.

2. Y. Asano. A New Framework for Link-based Information Retrieval from the Web. PhD thesis, The University of Tokyo, December 2002.

3. Y. Asano, H. Imai, M. Toyoda, and M. Kitsuregawa. Applying the site information to the information retrieval from the Web. In Proceedings of the 3rd International Conference on Web Information Systems Engineering, pages 83-92, 2002.

4. Y. Asano, H. Imai, M. Toyoda, and M. Kitsuregawa. Finding neighbor communities in the Web using an inter-site graph. In Proceedings of the 14th International Conference on Database and Expert Systems Applications, page to appear, 2003.

5. T. Munzer. Drawing large graphs with H3Viewer and site manager. In 6th International Symposium, GD98, LNCS1547, pages 384-393, 1998. 